\title{
Enantioselectivity investigation of short polar peptides with different positions in the Michael reaction
}

\begin{abstract}
This work reports the effectiveness of short polar peptides as asymmetric catalysts in Michael reactions to attain good yields of enantio- and diastereoselective isomers. In a comparison, glutamic acid and histidine produced greater ee and yields when they were applied as the second amino acid in short trimeric peptides. These short polar peptides were found to be efficient organocatalysts for the asymmetric Michael addition reaction in water.
\end{abstract}

Keyword: Asymmetric; Michael reaction; Organocatalyst; Short polar peptide. 